\title{
The genus Moenchia (Caryophyllacae) in Turkey
}

\author{
Mustafa KESKIN *1 \\ ORCID: 0000-0003-2454-1891
}

${ }^{1}$ Marmara University, Institute of Pure and Applied Sciences, Biology Program, 34722, Goztepe, Istanbul, Turkey

\begin{abstract}
A new identification key, full descriptions, and distribution records based on various herbaria of Turkish Moenchia are given in this study. Detailed and comparative descriptions of the studied species have been prepared. Also, a new species, Moenchia akmanii M.Keskin sp. nov., is described as for science. The new species is morphologically similar to $M$. graeca that is but differs by long stems, different leaves, non-flowering shoots, 3-nerved sepals, mauve anthers, etc. The new identification key has been created to distinguish taxa distributed in Turkey.
\end{abstract}

Keywords: Moenchia, Moenchia akmanii, new species, Turkey

\section{Türkiye'deki Moenchia (Caryophyllacae) cinsi}

\section{Özet}

Bu çalışmada, Türkiye'de yetişen Moenchia cinsine ait yeni teşhis anahtarı, betimlemeler ve dağılımlar çeşitli herbarium numunelerine dayanılarak verilmiştir. İncelenen türlere ait detaylı ve karşılaştırmalı betimlemeler hazırlanmıştır. Ayrıca Moenchia akmanii M.Keskin sp. nov. adıyla bilim dünyasına yeni bir tür tanıtılmıştır. Bu yeni tür, morfolojik olarak $M$. graeca türüne benzemekle beraber ondan uzun gövdesi, farklı yaprakları, çiçeksiz sürgünleri, 3-damarlı sepalleri, mor anterleri vb. karakterleri ile ayrılmaktadır. Türkiye'de yayılış gösteren taksonları ayırt etmek için yeni teşhis anahtarı oluşturulmuştur.

Anahtar kelimeler: Moenchia, Moenchia akmanii, yeni tür, Türkiye

\section{Introduction}

Moenchia is a small genus with only 3 to 5 species living mainly in the Mediterranean area [1]. It is certainly related to the genus Cerastium. In particular, it is separated from the genus Cerastium with non-disintegrating petals.

Four species of Moenchia have been reported in A Checklist of the Flora of Turkey [2]. After examining much-related literature $[1,2,3,4,5,6,7,8]$ about this genus in detail, an unnamed specimen is decided to be a new species and proposed here as Moenchia akmanii M.Keskin.

\section{Materials and methods}

During the revision of the genus Cerastium for Illustrated Turkish Flora, the genus Moenchia, which is closely related to this genus, was also studied. During this working time, all the specimens of Moenchia genus present in various herbaria (E, AEF, HUB, ISTE, ISTF, ISTO, MARE, MUFE, ANK, NGBB, VANF, IZEF - These herbarium samples were examined by using only the high-resolution pictures obtained on the website.-) was examined morphologically. The findings obtained during the examinations were comparatively studied. As a result, detailed

\footnotetext{
* Corresponding author / Haberleşmeden sorumlu yazar: Tel.: +905067638790; Fax.: +902167773201; E-mail: trifolium@ @otmail.com

(C) Copyright 2021 by Biological Diversity and Conservation $\quad$ Received: 02.07.2020; Published: 15.04.2021
} 
descriptions and distributions were prepared for all species and mapped. Furthermore, an unidentified specimen is described as a new species to science from Central Anatolia (Ankara province, Turkey).

\section{Results}

\subsection{Description of the genus Moenchia}

Moenchia Ehrhart, Beitr. Naturk. 2: 177 (1778). (nom. cons.), non Medik., non Roth.

Type species: Moechia erecta (=Moenchia quarternella Ehrh. (as Mönchia quarternella)).

Syn.: Alsinella Moench., Meth. 222 (1794) (non Hill 1756). Doerriena Borkh., Rhein. Mag. 1: 528 (1793).

Annual herbs. Stems glabrous, thin, ascending to erect, simple or sometimes branched. Cauline leaves connate, sessile or sometimes little petiolate in basal leaves; blade 1-veined, linear to linear-oblanceolate, apex acute or subacute. Inflorescence terminal, 1-10 flowered, spreading cymes or flowers solitary; bracts paired, subfoliaceous with scarious margins. Pedicels erect or subpatent in fruit. Sepals 4 or 5, free, green, usually linear-lanceolate, herbaceous, scarious, apex acute; petals 4 or 5, white or blue-mauve, blade apex generally entire or rarely small-dentate; stamens 4 , 8 or 10; styles 4 or 5; filaments longer than anthers; anthers generally ovate to spherical; stigmas 4 or 5; ovary ovate to subcylindrical and longer than or equal to stillus. Capsules cylindrical, opening by 8 or 10 revolute teeth, shorter than or equaling sepals. Seeds small, more, brown, tuberculate to submuricate.

\subsection{The New Identification key for Moenchia in Turkey}

1. Sepal and petal 4

2. Stamen 4 5b. M. erecta var. octandra

2. Stamen 8 ... 5a. M. erecta var. erecta

1. Sepal and petal 5

3. Petal blue-mauve 2. M. coerulea

3. Petal white

4. Petal longer than sepal 1. M. mantica

4. Petal at most equal to sepal

5. Stems 2-15 cm; sepal 1-nerved; anthers yellow. 4. M. graeca

5. Stems 15-25 cm; sepal 3-nerved; anthers mauve 3. M. akmanii

\subsection{The Checklist of Moenchia in Turkey with a New Species}

\subsubsection{Moenchia mantica (L.) Bartl. Cat. Hort. Gött. 5 (1839). [Fig.1, 2]}

Type: Described from Verona, Italy (Hb. Linn. 603/28, photo!). 13: 40 (1893).

Syn.: Cerastium manticum L., Sp. Pl. Ed.2, 629 (1762). M. dolichotheca Somm. \& Lev. in Act. Hort. Petrop.

Annual herbs. Stems ascending to erect, mostly dichotomous branched, 10-40 cm. Leaves 15-20 x 3-5 mm, glabrous, lanceolate to very narrowly elliptic-lanceolate, acute rarely obtuse. Inflorescence strict, usually manyflowered. Bracts ovate to lanceolate with broad scarious margins. Pedicels unequal, longest pedicel 45-70 mm. Sepals 5-parted, 6-9 mm, broad and lanceolate, elevated 1-veined, and c.10 thin vein. Petals white, 5-parted, slightly $2 \mathrm{x}$ longer than the sepals. Stamens 10, anthers yellow. Capsule ovoid, at most equal to sepal. Seeds 0.8-1.1 mm.

Examined specimens

Ağrı: Patnos, Top dağı, Kızkapan köyü üstleri, step, 390 17' 350"N ve 420 38' 200"E, 1850m, 17 v 2006, H.Emlik 1616 (VANF 3642!).

Afyonkarahisar: Bayat, Otlugedik sırtı kuzeyi, 1360m, 22 v 1975, M.Vural (GAZI 197!). Bolvadin, Emirdağ, Gedik yaylası, 1600m, 8 vi 1987, Y.Akman (ANK 14354!). Kocatepe, kıraç sırtlar, 22 v 1966, A.BAytop, B.Çubukçu (ISTE 9392!).

Ankara: Beypazarı, Alemdar köyü üstü, 1400m, 1 vii 1971 (ANK 788!). Beypazarı, Cuma deresi, köprübaşı, 2 vii 1972, Y.Akman (ANK 8516!). Beypazarı, Deliyar-Cuma deresi, Pinus nigra orman1, 1400m, 22 vii 1972, Y.Akman (ANK 8519!). Beypazarı, Elmabeli, Pinus nigra orman açıklığı, 700m, 27 v 1971 Y.Akman (ANK 788-A!). Beypazarı, Karaşaraltı, 1000m, 28 v 1971, Y.Akman (ANK 788-B!). Beypazarı, köprü yanı, 28 v 1972, Y.Akman (ANK 8520!). Beypazarı, Nallıkaş, nemli çayırlar, 1500m, 26 vi 1973, Y.Akman (ANK 8517!). Elmadağ, 1600m, 25 vi 1932, W.Kotte (ANK 122!). Kızılcahamam, Karaçam ormanı, 26 vi 1960, A.Huber-Morath (ANK!). Kızılcahamam, Soğuksu Milli park1, yanık sirtı, 1300-1350m, 12 v 1990, Ö.F. (GAZI 1328!). 
Aydın: Çine çayı vadisi, 21 iv 1969, K.Fitz (EGE 3973!). Çine çayı kenarı, 21 iv 1969, H.Güner (EGE 15184!). Dalaman-Cumalı 6.6km, çalılık yamaçta, 24 iv 1992, A.Byfield B 49 (ISTE 64875!).

Balıkesir: Ayvalık güneyi, Yassı tepe, 150m, 28 v 1997, K.Alpınar (ISTE 73953!). Ayvalık, Hakkıbey yarımadası, Cennet koyuna bakan yamaçlar, 15 iv 1998, K.Alpınar (ISTE 74992!). Edremit, Bursalıhanı, Pinus brutia ormanı, $10 \mathrm{v}$ 1966, H.Peşmen (EGE 15192!). Edremit, Kumluca, Eybek kule yolu, bir çoğu bir arada, bol miktarda, güney, 940m, 23 iv 1999, M.Tokcan (ISTO 28525!). Erdek, Marmara adası, maki ve çayır, 2 v 1965, E.Demiriz ((ISTF 5203!). Kozak, Kıranlı köyü, Madran çayı, 15 v 1966, H.Peşmen 565 (EGE 5885!). Marmara adası, Marmara yakınları, kireç ocakları üstündeki tepeler, 8 v 1971, A.Baytop, G.Ertem, F.Öktem (ISTE 19636!). Marmara adası, Nahiye merkezi, Çınarlıköy arasındaki yamaçlar, 100m, 6 v 1978, E.Tuzlacı (ISTE 39174!). Marmara adası, Pteridium altında, 550m, 17 vi 1968, A.Baytop, T.Avcigil (E ! ve ISTE 13740!).

Bilecik: Bozöyük-İnegöl, c. 37 km from İnegöl and c.1km from river Aksu to Bözöyük, N of the main road. Fairy steep slope, facing S, with open deciduous forest affected by grazing. 830m, 21 v 1990, Raino Lampinen 7660 (EGE 33879 !). Bitlis: Tatlıkaynak köyü, step, 1550m, 18 v 2002, A.Altık 2286 (VANF 3709!). Bitlis'in 5km kuzeyi, sulu çayırlar, 1350m, 18 v 1966, Davis 43139 (E !). Tatvan ile Küçüksu arası, 1750m, 19 v 1966, Davis 43293 (E !).

Bolu: Abant girişi, 840m, 25 v 1980, Y.Akman, E.Yurdakulol (ANK 10557). Abant, 1350m, 26 vi 1975, A.Baytop (ISTE 32276). Abant, 5 vii 1975, A.ve T.Baytop (ISTE 32405a!). Abant, Alpagut'u geçince Carpinus betulus ormanı, 1000m, 10 vi 1978, Y.Akman (ANK 9449!). Bandırma, Şahinburgaz, maki zonu, 300m, 19 v 1976, Y.Akman (ANK!). Bolu'ya inişte, Kuzey yamaçları, meşe ormanı, 800m, 10 vi 1980, Y.Akman, E. Yurdakulol (ANK 10558!). Gerede, Aktaş ormanları, Aşağı ovacık, Pinus sylvestris katı, 1400m, 3 vii 1975, O.Ketenoğlu (ANK 428!). Gerede-Aktaş ormanları, sarıçam katı, 1400m, 3 vii 1975, O.Ketenoğlu (ANK 428!). Bolu işletmesi, 14/00, Kökez bölgesi, Sultan serisi, bölme no: 38, bak1 N, 930m, 16 vii 1978, H.Aksoy 371, (ISTO 25661!). Bolu işletmesi, 14/00, Kökez bölgesi, Sultan serisi, bölme no: 44, bakı SSE, 970m, 9 vi 1978, H.Aksoy 58, (ISTO 25573!). Köroğlu, Karaçamaltı, anakaya volkanik, 1400m, 26 vi 1975, Y.Akman (6310!). Sarı Mestan, 27 vi 1997 Y.Akman (ANK!). Dolubaba-Yiğitali, 14 v 1955, B.Tutel ve M.Heilbron (ISTF 14129!).

Bursa: Işıklar köyü, 5 v 1950, T.Baytop, A.Berk (ISTE 297!). Uludağ, Soğukpınar, 500-1000m, 15 v 1962, Dudley 34751 (E!). Uludağ, 27 iv 1883, P.Sintenis (E !). Uludağ, 17 v 1899, J.Bornmüller 4210 (E !). Uludağ, orman zonu, 1450m, ix 1964, F.F.E.De Wilde (E !). Uludağ, Softaboğan'nın batısı, Abietum, 1700m, 10 viii 1951, H.Demiriz (E !).

Çanakkale: Gökçeada, Bademli köyü üstü, Yıldız tepesi kuzeyi, 0m, 16 iv 1977, Ö.Seçmen, E.Leblebici (EGE 20346!). Gökçeada, Değirmendere Ketveren tepesi, kuzey doğusu, 30m, 19 v 1975, Ö.Seçmen, E.Leblebici (EGE 23039!). Gökçeada, Pınarbaşı-Kaleköy arası, vadi içinden, 100m, 18 iv 1977, Ö.Seçmen, E.Leblebici (EGE 20345!). Gökçeada, Yıldız köyü Kuzulimanı arası, 15m, 14 iv 1975, Ö.Seçmen, E.Leblebici (EGE 23038!). Gökçeada, Yıldız köyü Kuzulimanı arası, 20m, 14 iv 1975, Ö.Seçmen, E.Leblebici (EGE 23040!). Kurudağ, 28 iv 1968, A. ve T. Baytop (ISTE 12560!). Yenice, Kalkım Ayı gediği yolu, Karaçam altında bol miktarda, 840m, 16 v 1999, M.Tokcan (ISTO 28526!).

Çankırı: Atkaracalar, Dumanlıdağı, Avlağa-Sivren yaylası arası, step, 1500-1600m, 9 vii 1992, Ahmet Duran (GAZI 1572!). Işık dağı, Karaçam altı, 1400m, 12 vi 1975, Y.Akman (ANK 6317!). Saka dağı, yayla, 1050m, 27 vi 1980, M.Demirörs (ANK 172!).

Denizli: Babadağ, Babadağ ilçeden Başalan yaylasına çıkarken $2 \mathrm{~km}$, yamaçlar, 1300m, 9 v 1986, S.Oluk, Ö.Seçmen, Y.Gemici (EGE 34863!). Babadağ, Gökbel yaylası, Acılık tepesi, eğimli arazi, 1750m, 17 v 1986, S.Oluk (EGE 34869!). Buldan, 29 vi 1965, C.Regel (EGE 15183!). Çivril, Sığırkuyruğu yaylası, 1300-1400m, 4 v 1983, Y.Gemici (EGE 25459!). Honaz dağı, Başalan mevkii, yol kenarı, 700m, 17 v 1973, E.Tuzlacı (ISTE 24762!). Honaz dağ1, Döngelli tepeden Honaz'a inen oduncu yolu, 950m, 18 v 1973, E.Tuzlacı (ISTE 24851!). Honazdağ1, Baymanlı mevkii, 700m, 18 v 1973 (ISTE 24867!).

Edirne: Babaeski-Havsa arası, Havsa yakınları, Simit reverzuarına iniş yolu, 8 v 1973, A.Baytop, E.Tuzlacı (ISTE 24402!). Keşan-Enez yolu, Kılıçköy'den 1,5km önce, merada otlu çukurlar ve tepecikler üzerinde, 18 iv 1992 , A.Byfield B 26 (ISTE 64852!). Keşan-İpsala yolu, İpsala yakınları, yol kenarı, 22 iv 1970, A.Baytop, F.Öktem (ISTE 17658!).

Eskişehir: Sündiken dağ, Kalabak deresi, 1400-1600m, 12 vi 1971, T.Ekim (E ! ve ANK 335!). Türkmen dağ1, Kazan çukuru, 1400m, 16 vi 1976, T.Ekim (ANK 2128!).

Giresun: Merkez, Ülper köyü, yumuşak toprak üzerinden, 1200m, 23 v 1983, Ö.Yeşilada (HUB 2837!).

Ĭgdır: Tuzluca, Hadımlı-Sarıabdal arası tepeler, 1279m, 30 v 2007, E.Altundağ (ISTE 84538!).

İstanbul: Alemdağ, 18 iv 1952, T.Baytop, A.Berk (ISTE 3005!). Anadolu hisarı, 19 v 1942, M.Başarman (ANK!). Ataşehir: Nezahat Gökyiğit Botanik Bahçesi, doğal alan bitkileri koleksiyonu, tarihsiz, M.Vural (NGBB 006115!). Aydos yolu, Aydos dağı, Hayrettin çeşmesi, maki, 12 v 1958, B.Tutel (ISTF 16982!). Aydos, 1 iv 1951, A.Berk (ISTE 3690!). Aydos, 10 v 1950, T.Baytop, A.Berk (ISTE 3716!). Aydos, 22 iv 1951, A.Baytop (ISTE 3710 !). Aydos, 3 iv 1952, T.Baytop, A.Berk (ISTE 3682!). Aydos, 3 iv 1952, T.Baytop, A.Berk (ISTE 3705!). Aydos, Yakacik, 24 iv 1966 , N.Tanker (ISTE 8687!). Beykoz, Dereseki, Karakulak suyu çevresi, ormanaltı, 11 v 1967, H.Demiriz, B.Tutel, A.Aydın (ISTF 22079!). Büyükdere, 30 iv 1956, E.Leblebici (EGE 15193!). Çatalca, Arnavutköy girişi, set üstü, 03 v 1989 , E.Üzen (ISTF 35820!). Çatalca, Hadımköy, Çanakça, Çanakça-Kestanelik, yol kenarı, 70m, 12 iv 2002, İ.Genç (ISTE 82065!). Çatalca, Hadımköy, Tugay, sevgi korusu, 60m, 28 iv 2002, İ.Genç (ISTE 82094!). Çatalca, mezarlık yakınları, 
1 v 1964, A. ve T.Baytop (ISTE 7605!). Çatalca, Subaşı köyü çevresi, buğday tarlası kenarı, 24 v 1972, N.Özhatay, E.Tuzlacı (ISTE 21880!). Çatalca, Subaşı yolu, 21 v 1974, A.Baytop, E.Tuzlacı (ISTE 28164!). Çatalca, Subaşı, Piknik alanı, yol kenarı, 80m, 19 v 2002, İ.Genç (ISTE 82137!). Çekmeköy, Çataltepe Kalebayırı yolu sulak nemli sazlıklar, 254m, 23 iv 2016, M.Keskin, E.Özhatay, N.Özhatay !. Ispartakule, Kapadık çiftliği, 14 v 1949, M.Başarman (ISTF 8552!). Kanarya, Soğuksu, 31 v 1964, A. ve T.Baytop (ISTE 7672!). Karaburun-Terkos aras1, 2km, 13 v 1962 , Betül Tutel (ISTF 18480!). Kayışdağ, 16 iv 1952, T.Baytop, A.Berk (ISTE 3704!). Kemerburgaz, Ağaçl1, 0,5km, S., çayır, 4 v 1968, H.Demiriz (ISTF 23065!). Kilyos, sol taraftaki sahil tepeleri, 22 v 1992, Ş.Şiraneci, E.Akalın (ISTE 63924!). Küçükbakkalköy-Kayışdağ1 yolu üzerinde, 12 v 1963, Betül Tutel (ISTF 18847!). Küçüksu, 22 iv 1966, A.Ali, E.Günhan, S.Türkseven (ISTE 8635!). Maltepe, Başıüyük Mahallesi, 7 ii 2001, M.Keskin 2462 (ISTE 79290 !). Maltepe, Cevizli, Dragos tepesi, N yamaçlar, çayır, 28 iv 1968, A.Çırpıcı, O.Sunter (ISTF 23035!). Maltepe, Cevizli, İzmit yolu, çayır, 11 iv 1971, A.Çırpıcı (ISTF 25140!). Olimpiyat köyü alanı, vadinin olimpiyat sahası tarafı, harfiyat yapılan alanlarının altları, taşlık alanlar, 6 v 2001, E.Akalın, U.Uruşak (ISTE 79932!). Maltepe: Cevizli-Dragos tepenin NE yamaçları, 14 v 1971, E.Özhatay 282 (ISTO 18653!). Pendik, Akfirat belediyesi, 25 iv 2005, M.Keskin 3765!. Pendik, Akfırat-Göçbeyli, Aydınlık sokak girişi, giriş yolunun solundaki Taşlı makilikler, 105m, 13 v 2017, M.Keskin 2161, N.Özhatay, E.Özhatay !. Sancaktepe, Turkuaz su fabrikası yakınları, Fabrikalar alanı, çayır, 15 iv 2017, M.Keskin 6813!. Sarıyer, Hacıosman bayırı, yamlı çayır, 15 v 1973, Tutel, Çırpıcı, Kuş (ISTF 27116!). Sarıyer: Belgrad ormanı, Balaban dere, 28 iv 1959, F.Yaltırık (E ! ve ISTO 1317!). Sarıyer, Belgrad ormanı, 1951, İ.Akbaş (ISTO 148!). Silahtarağa ilerisindeki tepeler, 13 v 1967, N. ve M.Tanker (ISTE 11205!). Silivri, Danamandıra, Tabiat Parkı yolu-Sinekli yolu ayrımı, gençleştirilmiş meşe ormanı, M.Keskin 6220, N.Özhatay, M.Özhatay !. Silivri, Gelevri, Gülçin çiftliği, bodur orman, Quercus, Phillyrea, Carpinus, kurak çayır, 16 iv 1961, H.Demiriz (ISTF 17892!). Silivri, Lüleburgaz deniz evleri civarı, G.Ertem, 30 iv 1973 (ISTE 24252!). Terkos fidanlığı yakını, 3 v 1971, A.Baytop, G.Ertem, N.Özhatay, (ISTE 19478!). Terkos yolu, Çınarlıhan civarı, uzunca ova, makide, 150m, 12 v 1951, H.Demiriz (E ! ve ISTF 11078!). Terkos yolu, Habibler köyünde 2km önce, maki, 13 v 1962, B.Tutel (ISTF 18460!). Terkos, Fidanlık yolu kenarı, 3 v 1971, Ertem, E.Özhatay 264 (ISTO 18638!). Rumeli hisar1, 20 v 1918, B.V.D.Post (E !).

İzmir: Bergama, Kozak, Kıranlı odun deposu, 750m, 24 iv 1986, Ö.Seçmen (EGE 17938!). Kemalpaşa-Sincancılar, Ovacık, 300m, 12 v 1969, G.Oğuz (EGE 15187!). Ödemiş, Bozdağ, 29 v 1962, C.Regel (EGE 15185!). Ödemiş, Keldağ1, kayalık yerler, 1350m, 31 v 1980, E.Tuzlacı, T.Çelebioğlu (ISTE 44382!). Yamanlar dağ1, 24 v 1995 , K.Alpınar, G.'t Hart (ISTE 67943!).

Kastamonu: Ilgaz dağları, Kanlı göl, Çeşme üstü, 1300m, 13 vi 1982, Y.Akman, E.Yurdakulol, M.Demirörs (ANK 12118!). Ballıdağ, Sarıçam ormanı, 1350m, 11 vi 1979, O.Ketenoğlu 881 (ISTO 25187!). Daday arası, Sarıoluk, meşelik, 1000, 19 vi 1978, O.Ketenoğlu (ANK 880!). Daday arası, taş ocakları, 950m, 23 vi 1980, O.Ketenoğlu (ANK 883!). Daday, Kiraz serisi, Abies ormanı, 1100m, 19 vi 1978, O.Ketenoğlu (ISTO 29468!). Devrekani, Oyrak II geçidi, Pinus sylvestris topluluğu, anakaya ofiolitik, 1210m, 08 vi 1991, E.Yurdakulol (ANK 3517!). Tosya, Ahlatdağı bölgesi, Büyükdere serisi, Dikmen tepe mevkii, Pinus nigra ormanı 1500m, 11 vi 1975, M.Kılınç (ANK 1085!). Tosya, Ortalıca yaylası, -Acıkavak yaylası arası, Karaçam ormanı, 1350m, 30 vi 1976, M.Kılınç (ANK 5069!).

Kırklareli: Demirköy, Demirköy dökümhanesi, 176m, 30 v 2009, N. ve E.Özhatay, E.Akalın, S.Demirci (ISTE 91904!). Demirköy, Saka longosu, 28 iv 1974, N. ve E. Özhatay (ISTE 27690!). Demirköy, Sarpdere yolu, $511 \mathrm{~m}, 14$ vi 2009, E.Akalın \& S.Demirci (ISTE 92080!). Dereköy-Hudut arası 4km, yol kenarı, otlu alanlar, 525m, 23 v 2009 , N.Güler, H.Ersoy (ISTE 96220!). Pınarhisar'dan 9 km, çalılıklar arası, 21 v 1974, A.Baytop, E.Tuzlacı (ISTE 28226 !). Üsküp yolu, ana kavşaktan c.4km, sirtlarda, 21 v 1974, A.Baytop, E.Tuzlacı (ISTE 28241!). Eski Saray yolu, tarlalardan aşağıya inerken, 40m, 21 iv 2005, D.Oral 1521 (ISTO 33579!).

Kocaeli: Gebze-İzmit yolu, 2. km, yol kenarı, 14 v 1970, E.Özhatay 128 (ISTO 18498!).

Kütahya: Azant'tan Pehlivan taşına, Eğrigöz 3km geçince, 1360m, 8 vi 1978, G.Görk (EGE 37882!). Domaniç-İnegöl arası, Duraköy ilerisi, 940m, 11 v 1982, K.Alpınar (ISTE 48659!). Emet, Eynal'dan Emet'e doğru 9km sonra, açık habitat, kumlu fiğ habitatı, 1200m, 17 v 1978, Ö.Seçmen, G.Görk (EGE 37883!). Emet, Yeniceköy'den arpa tarlasına giden yolda, geniş çayırlıkta, çeşmelerin yanında, 1100m, 08 v 1980, G.Görk (EGE 18347!). Gediz, Murat dağ1, Çukurören, çayır, 1100m, 16 v 1980, A.Çırpıcı (ISTF 34088!). Gediz, Murat dağı, Hamam yukarısı, göl yeri, orman arası açıklık, 1750m, 9 vi 1977, A.Çırpıcı (ISTF 30468!). Gediz, Murat dağı, İkizce, çayır, 1450m, 21 vi 1978, A.Çırpıcı (ISTF 32033!). Gediz, Muratdağı, Hamam Çukurören köyü arası, Yayla çayırlık Pinus nigra orman açıklığı, 1500m, 3 vii 1972, S.Oflaz, G.Oğuz, Ö.Seçmen, E.Leblebici (EGE 15375!). Gediz-Uşak, 55km Uşak'ın 10 km kuzeyi, Cistus laurifolius topluluğu, 1140m, 14 vi 1954, H.Demiriz (E ! ve ISTF 13322!). Hamam-Sobalar, çayır, 1450m, 17 v 1978, A.Çırpıcı (ISTF 31454!). İnönü-Kütahya arası, kurak tepeler, 1000m, 21 v 1966, A.Baytop, B.Çubukçu (ISTE 9347!). Simav, Kiçir-Akdağ, Pinus nigra ormanı, 1700m, 19 vi 1965, M.J.E.Coode ve B.M.G.Jones (E !, ISTF 20901!, ISTO 6712!). Simav'dan yaklaşık 10km, orman yolundan Simav dağına giderken, 1500-1700m, 18 vi 1965, M.J.E.Coode ve B.M.G.Jones (ISTF 20881! ve ISTO 6741!). Simav-Demirel aras1, Demirci'den 11 km, Pinus nigra orman alt1, 1350m, 9 vi 1972, S.Oflas, G.Oğuz, Ö.Seçmen, E.Leblebici (EGE 15186!). Murat dağ1, Gediz üstleri, Kesik Söğüt, 1200m, 5 vii 1962, Davis 36723 ve Coode (E !). Simav, dağ yolu, 1500-1700m, 18 vi 1965, M.J.E.Coode ve B.M.G.Jones (E !).

Manisa: Akhisar, Kertil mevkii, Pinus brutia orman1, 23 iv 1973, Öner, Seçmen, Ahmet (EGE 11391!). AkhisarGölmarmara, step, 29 iv 1966, H.Peşmen (EGE 4862!). Manisa dağı, 1500m, 13 vi 1966, A.Baytop, B.Çubukçu, 
T.Avcıgil (ISTE 9981!). Manisa dağı, 3 v 1961, Turhan Baytop (ISTE 6428!). Manisa dağı, 4 iv 1966, N.Zeybek, C.Regel (EGE 15189!). Manisa dağ 1 , Karlık kuzeyi, 1140m, 25 v 1984, H.Duman (GAZI 1075!). Manisa dağı, yamacı, 700m, 4 iv 1966, C.Regel, H.Peşmen, G.Oğuz (EGE 15191!). Soma, Adilköy, kuzeydoğusu, Bahçecik çeşmesi üstü, 750m, 18 v 1967, H.Peşmen, M.Aydar (EGE 15190!). Soma, Madenci çeşmesi yol kenar1, 600m, 12 v 1977, Ö.Seçmen (EGE 23753!). Çine arası, kayalık habitat, 28 iii 1979, Ö.Seçmen, Leblebici, Bekat (EGE 17273!).

Muğla: Karaçam ormanı altı, 1200-1350m, 18 vi 1976, Y.Akman ve Quezel (ANK 7290!); Yatağan-Gökbel, Quercus coccifera makisi, 300m, 20 iv 1965, Dr. Davis 41456 (ISTO 5863!).

Samsun: Kurupelit kampüsü, Fen-Edebiyat Fakültesi çevresi, 4 v 1995, M.Keskin 153 (ISTE 77071!). Samsun'un 8km doğusu, çayırlık, 19 v 1963, Carl Tobey 120 (E ! ve ISTO 1926!). Samsun'un 5km doğusu, Karadağ, 50m, 15 vi 1963 , Carl Tobey 341 (E ! ve ISTO 2014!). Kizılırmak, Asar'dan sonra, 450m, 12 v 1967, Tobey 1782 (E !). Hac1 İsmail köy, açık alanlar, 300m, 6 v 1967, Tobey 1733 (E !). Derbent, Ökçetepe'nin kuzey yamacı, Asarağaç tepe, 30-40m, 22 v 1952, H.Demiriz (E !).

Sinop: İnce burun, volkanik arazi, 30m, 23 iv 1966, Tobey 1621 (E !).

Sivas: Zara, 20 km güneyi, jipsli arazi, 1500m, 6 vii 1986, B. Yıldız (ISTE 94275!).

Tekirdağ: Keşan, Malkara arası, 19 v 1970, A.Baytop, F.Öktem (ISTE 17974a). Kırkgöz köyü, Londra asfaltı, kavaklık alt1, 30 iv 1973, G.Ertem (ISTE 24253!). Malkara-Keşan yolu, Pişman koruluğu, 20km içeride, 12 v 1991, E.Akalın (ISTE 62892!). Seymen-Çorlu arası, Çorlu'ya 2km kala, 22 iv 1970, A.Baytop, F.Öktem (ISTE 17635!). Seymen-Çorlu aras1, Çorlu'ya 7km kala, 22 iv 1970, A.Baytop, F.Öktem (ISTE 17619!).

Trabzon: Vakfıkebir, Fol köyü, çayırlık, 1500m, 11 vii 1986, Y.Gemici (EGE 30162!).

Van: Özalp, Dorutay köyü batı kesimi, step, 2658m, 25 vi 1996, F.Özgökçe 2654 (VANF 3625!). Özalp, Damlacık köyü doğusu, Boncuk deresi vadisi, step, 2150m, 9 vii 1997, F.Özgökçe 5730 (VANF 3638!). Muradiye, Alkasnak köyü, Ziyaret tepesi batısı, nemli, çayırlık alan, 1900m, 12 vi 2001, O.Karabacak 1698 (VANF 3708!). Gürpınar, Hamurkesen köyünün arkaları, step, 2000m, 22 v 2002, M.Ünal 6760b (VANF 3779!). Gürpınar, Hamurkesen köyünün arkalar1, step, 2000m, 22 v 2002, M.Ünal 6814 (VANF 3780!).

Yalova: Orhangazi yolu, 3 v 1950, A.Berk, T.Baytop (ISTE 2474!).

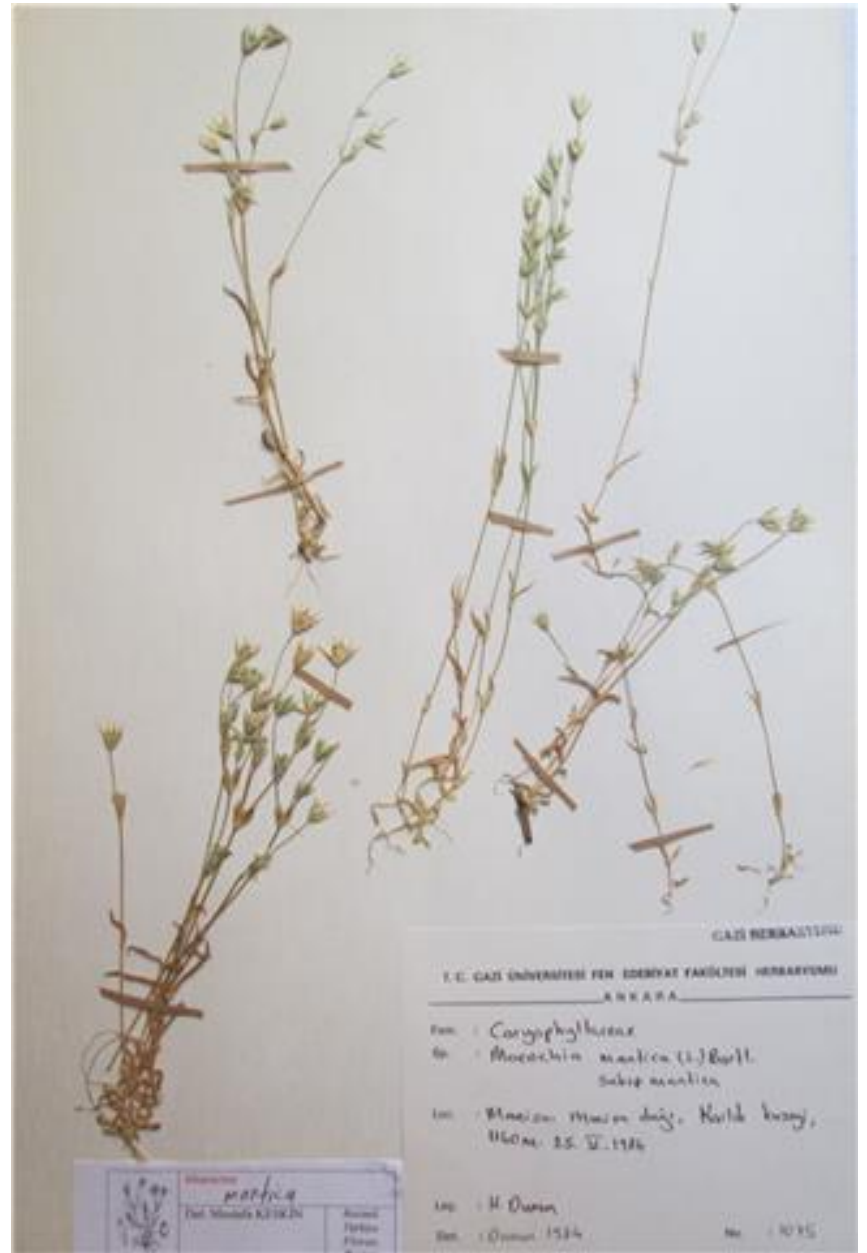

Figure 1. A Herbarium sheet

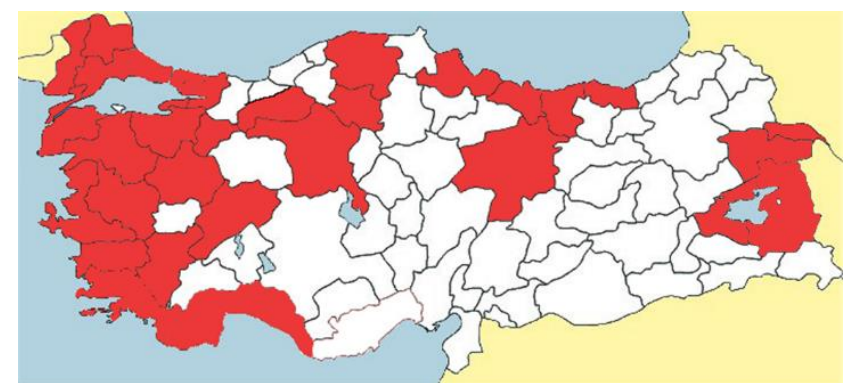

Figure 2. Distribution map 
It is probably the most widely distributed and is available in many parts of Turkey. It is the most basic member of slightly moist meadows with white flowers, especially in early spring. M. mantica is a very characteristic species due to its double-length petals of calyx, its upright posture, and its early nature compared to other species.

\subsubsection{Moenchia coerulea Boiss., Fl. Orient. 1: 712 (1867). [Fig. 3, 4]}

Syn.: Cerastium coeruleum Boiss., Diagn. Ser. 1(1): 53 (1853). M. mantica L. var. violascens Azn., Bull. Soc. Bot. Fr. 44: 166 (1897). M. mantica L. forma coerulea (Boiss.) Janchen, Zwei für Österreich neue Pflanzen, 5 : 59 (1907). M. mantica L. var. jolanthae Kovâts, Ungarische Botanische Blätter - 29: 49 (1930).

Holotype: Montes Smyrne, Mai 1842, Boissier (E !).

Annual herbs. Stems usually strict, simple or branched from base, dichotomous branched at upper, 10-20 cm. Leaves 7-15 x 2-4 mm, glabrous, linear-lanceolate, acute. Inflorescence strict, 2-7 flowered. Bracts broad ovatelanceolate with broad scarious margin. Pedicels unequal, longest pedicel 40-75 mm. Sepals 5-parted, 6-9 mm, oblonglanceolate, mauve at upper, usually veins obscure, main vein sub elevated. Petals blue-mauve, 5-parted, longer than the sepals, usually not 2 x calyx white. Stamens 10, anthers yellow. Capsule subovoid, exceeding to sepals.

Examined specimens

Afyonkarahisar: Bayat, Bağlik mevkii üzeri, 1120m, 23 v 1975, Mecit Vural (ANK 198!).

Antalya: Akseki-Bademli arası, Toy alanı, step, 1400m, 25 v 1983, M.Koyuncu 6040-S.Erik (HUB 02879!).

Aydın: Eskiçine, Madrandağı, Topçam köyü güneyi, yamaçtan, 800m, 29 iv 1977, A.Yayıntaş, A.Yürül, A.Tamar; F.Seliayan (EGE 15180!).

Denizli: Çivril, Sorgun'dan-Kocayayla'ya çıkarken, 1250m, 5 v 1983, Y.Gemici (EGE 25460!). Sarayköy, BabadağHisarköy'den Tekçam'a doğru 7km, 1050m, 22 v 1972, G.Oğuz, Ö.Seçmen, E.Leblebici, T.Kesercioğlu (EGE 15181!).

Edirne: Merkez, Edirne'nin 8km batısı, 100m, 19 iv 1966, Davis 41971 (E !).

Elazı̆̆: Kup dağı, Sumalı yayla yeri, Quercus topluluğu, 1600m, 10 v 1982, H.Susen (ANK 1089!).

Isparta: Şarkikaraağaç, Kızıldağ milli parkı, Kolonkaya kuzey yamaç, 1200m, 11 v 1995, B.Mutlu 1351 (HUB 37178 ). Sütçüler, Kuzca köyü, Kuzcalan, Astragalus stebi, kırmızı toprak, 1350m, 5 vi 1975, H.Peşmen-A.Güner 2062 (HUB 02875!).

İstanbul: Arnavutköy, Durusu, İSKİ girişi, 70m, 19 v 2017, M.Keskin 7092, N.Özhatay, E.Özhatay!. Arnavutköy, Durusu kumul girişi, 8m, 19 v 2017, M.Keskin 7116, N.Özhatay, E.Özhatay !. Terkos gölü, Karaburun'dan Tahlisiye'ye doğru, kumluk yol kenarı, Quercus makiliği, 50-60m, 8 v 1962, Dudley 34535 (E !).

İzmir: Bornova, Kurudere, Karaçoban mevkii, 6 v 1971, M.Aydar, C.Ödemiş (EGE 15188!). Bornova: Kurudere köyü, Mengene mevkii, 6 v1971, Aydar Ödemiş, (EGE 11656!). Kemalpaşa, Nifdağ1, zirve yolu, yangın kulesine doğru, 1300m, 16 v 1975, Ö.Seçmen, E.Leblebici (EGE 24322!). Kemalpaşa, Nif dağ1, 600m, 27 iv 1987, N.Zeybek (IZEF 2703!). Kemalpaşa, Nif dağı, Manastır kalesi, 450m, 9 v 1992, N.Zeybek (IZEF 1652!). Ödemiş: N Birgi, $500 \mathrm{~m}, 17$ v 1993, E.Sauer (IZEF 1254!). Yamanların şark1 ve karaçamdaki kurak otlaklarda, 900-1200m, v.1932, O.Schwarz (EGE 23174!). Bozdağ: pinetum, 1400m, 5 vii 1968, Huber-Morath (ISTO 15095!). İkikardeş, 15 v 1906, J.Bornmüller 9157 (E !). İzmir dağları, vi 1842, E.Boissier (E !).

Muğla: Eskiçine'ye gelmeden, Çine çay1, 5 iv 1995, N.Keyikçi, A.Er, G.Görk (EGE 18984!). Muğla, Pinus brutia, 19 iv 1965, C.Regel (EGE 15182!). Yatağan-Çine arası, Gökbel mevkii, granit kayalıkları, 300m, 2 v 1982, T.Ekim ve M.Koyuncu 5152 (AEF!). Yatağan-Çine, Quercus coccifera çalılıkları arası,100m, 1 iv 1956, Davis 25553 et O.Polunin (E ! ve ANK!). Yatağan ile Gökbel arası, Quercus coccifera makiliği açıklıkları, 300m, 20 iv 1965, Davis 41456 (E !).

Samsun: Ladik istasyonu, Karadağ, 1050m, 8 v 1965, C.Tobey 948 (E !). 


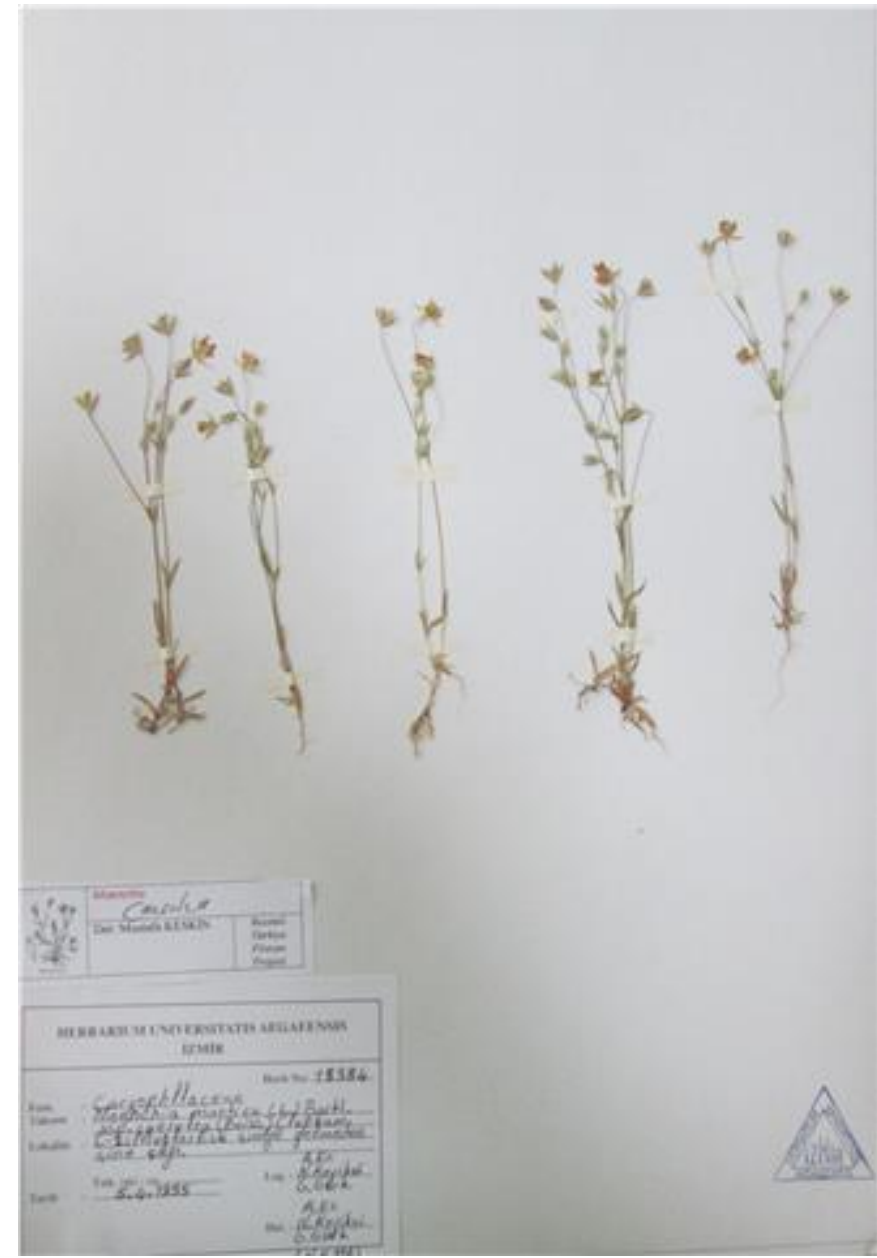

Figure 3. A Herbarium sheet

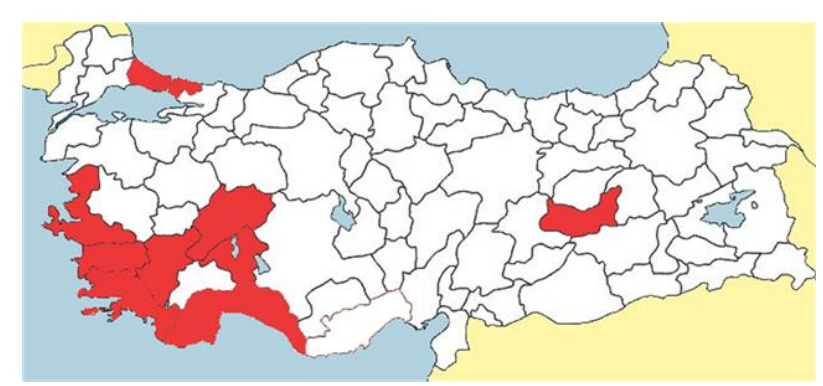

Figure 4. Distribution map

The most characteristic feature of the $M$. coerulea is the presence of blue to the purple corolla. This species is strictly related to $M$. mantica. But it is distinguished by the following characters:

1) Corolla blue to purple

2) Small, linear-lanceolate leaves

3) Corolla longer than calyx but never 2-fold

4) Capsule equal to sepals or slightly longer than sepal

\subsubsection{Moenchia akmanii M.Keskin sp. nov. [Fig. 5, 6]} (ANK 8518!)

Type material: Türkiye, Ankara: Beypazarı, Eğriova, yavaş akan dereciklerde, 1550m, 26 vi 1973, Y.Akman

Diagnose: Moenchia akmanii M.Keskin is close to M. graeca Boiss. \& Heldr. but differs from it by its long stems (short in M. graeca); cauline leaves 4-6 pairs, 20-35 x 1.0-2.0 mm (2-4 pairs, 4-14 x 1.5-2.5 mm in M. graeca); axillary shoots present (absent in $M$. graeca); flowers 4-9 (1-5 in M. graeca); 5-6 x 1.8-2 mm, caudate at anthesis and 3-nerved ( 7 × $3 \mathrm{~mm}$, acute at anthesis and 1-elevated nerved in M. graeca); anthers mauve (yellow in M. graeca).

Description: Annual herbs. Stems green, thin and flexible, 20-36 cm. Basal leaves linear, more or less withered at anthesis. Internodes very long. Cauline leaves 4-6 pairs, 20-35 x 1.0-2.0 mm, linear-elliptic, acute, not glaucous, 1-main vein. More axillary shoots present. Bracts $3-5 \mathrm{~mm}$, elliptic with broad scarious margins, midvein brownish, and first bracts fimbriate at the base. Inflorescences 4-9 flowered, axillary; each flower supported by two bracts. Flowering pedicels 10-15 mm; fruiting pedicels $30-40 \mathrm{~mm}, 5-7$ times as long as calyx, distinctly thickened above. Sepal 5; 5-6 x 1,8-2 mm at anthesis, narrowly lanceolate, with narrow scarious margins, caudate; 3 -nerved, not indurate, and decurrent 
to the base of calyx. Petal 5, white, as long as sepals or rarely exceeding, spatulate. Stamens 10; anthers mauve. The capsule as long as sepals (submature). Seeds c. $0,5 \mathrm{~mm}$.

Flowering time: June (probably to July).

Habitat: Slow flowing streams, meadows

Etymology: This new species named after Professor Yıldırım Akman who is a leading Ecologist, author, and collector of this sample.

Examined specimens:

Ankara: Beypazarı, Eğriova, Orman açıklığı, 36 T 0417361,4481291, 1598m, 12 vi 2014, Haşim Altınözlü, M.Erol Demirbilek (NGBB 004337!).

Notes: M. akmanii is probably only an endemic species of Eğriova/Beypazarı.

The proposed new species is strictly related to $M$. graeca. Differences are summarized in table 1 . Nevertheless, the first impression as a general structure seems to be the M. mantica type, but it is easily separated from the corolla as sepal or short, linear leaves, etc.

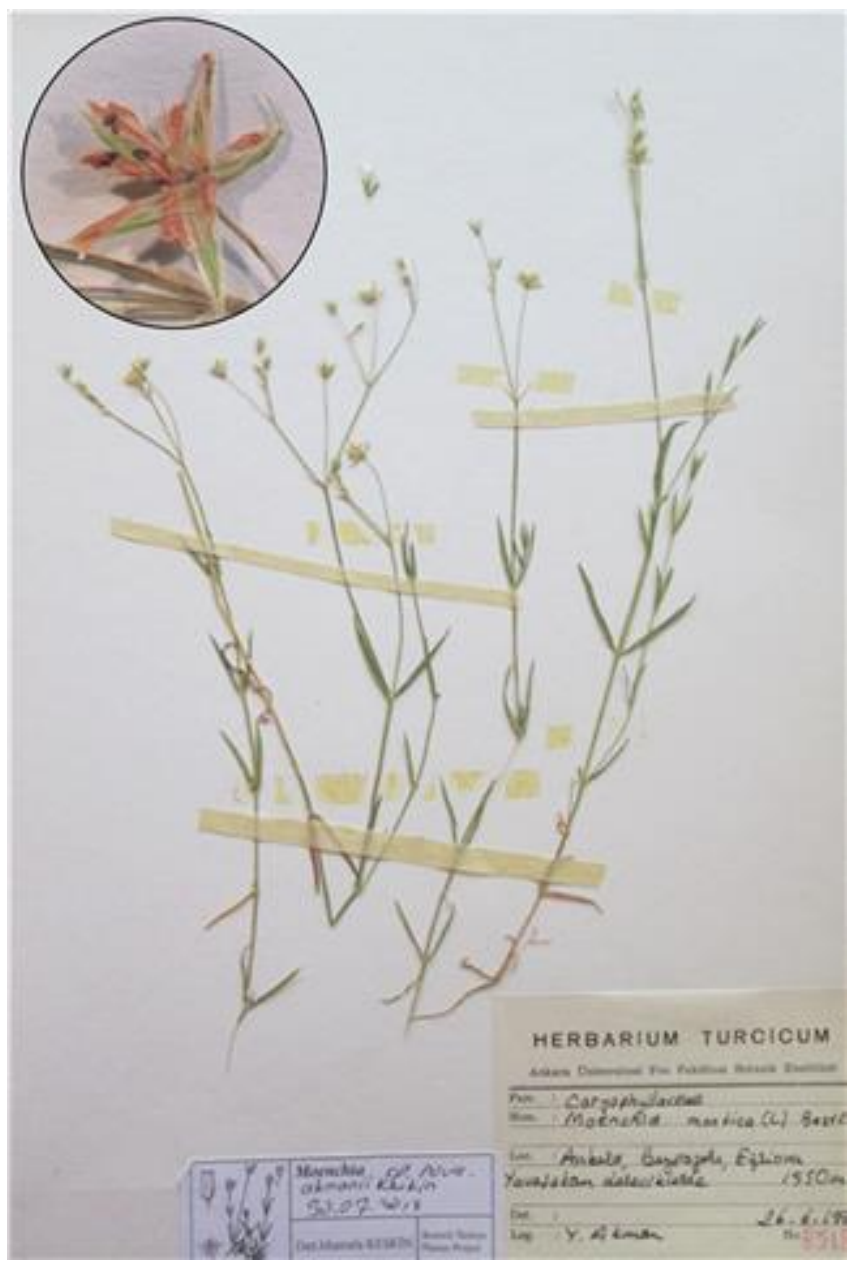

Figure 5. A Herbarium sheet (Holotype)

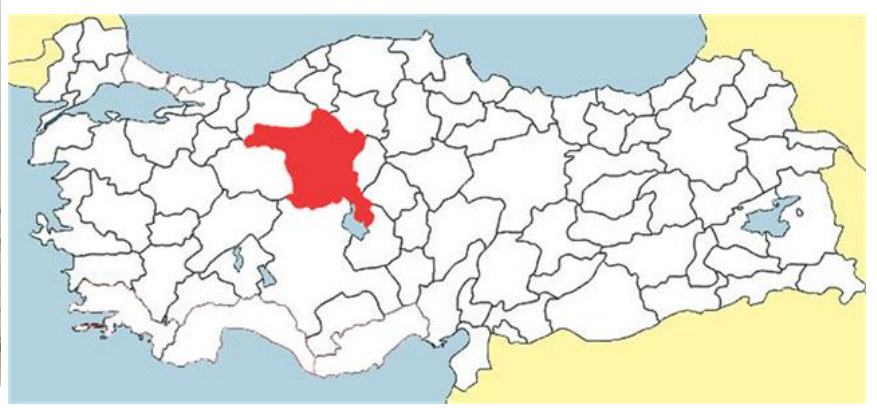

Figure 6. Distribution map 
Table 1: Differences between $M$. graeca and M. akmanii

\begin{tabular}{|c|c|c|c|}
\hline & graeca & mantica & akmanii \\
\hline Stem height & $4-15 \mathrm{~cm}$, tinged purple & $10-40 \mathrm{~cm}$ & $\begin{array}{l}20-36 \mathrm{~cm} \text {, green, somewhat } \\
\text { flexuous }\end{array}$ \\
\hline Basal leaves & $\begin{array}{l}\text { Oblanceolate, more or less } \\
\text { withered at anthesis. }\end{array}$ & $\begin{array}{l}\text { Linear to oblanceolate, more } \\
\text { or less withered at anthesis. }\end{array}$ & $\begin{array}{l}\text { linear, more or less withered } \\
\text { at anthesis }\end{array}$ \\
\hline Cauline leaves & $\begin{array}{l}\text { 2-4 pairs, } 4-14 \text { x } 1.5-2.5 \mathrm{~mm} \text {, } \\
\text { linear-elliptic, } \quad \text { subacute, } \\
\text { somewhat glaucous. }\end{array}$ & $\begin{array}{l}3-5 \text { pairs, } 15-20 \times 3-5 \mathrm{~mm} \text {, } \\
\text { glabrous, lanceolate to very } \\
\text { narrowly elliptic-lanceolate }\end{array}$ & $\begin{array}{l}\text { 4-6 pairs, } 20-35 \text { x } \mathbf{1 . 0 - 2 . 0} \\
\text { mm, linear-elliptic, acute, } \\
\text { not glaucous }\end{array}$ \\
\hline Axillary shoots & Absent & Absent & Present \\
\hline Bracts & $\begin{array}{l}\text { Elliptic with broad scarious } \\
\text { margins, midvein green }\end{array}$ & $\begin{array}{l}\text { Ovate to lanceolate with } \\
\text { broad scarious margins }\end{array}$ & $\begin{array}{l}\text { First bracts fimbriate at the } \\
\text { base, elliptic with broad } \\
\text { scarious margins, midvein } \\
\text { brownish }\end{array}$ \\
\hline Inflorescences & Apical & Apical & Axillary \\
\hline Flowers & $1-5$ & $3-8$ & 4-9 \\
\hline Fruiting pedicels & $\begin{array}{l}2-8 \text { times as long as calyx, } \\
\text { distinctly thickened above. }\end{array}$ & $\begin{array}{l}\text { 3-7 times as long as calyx, } \\
\text { distinctly thickened above. }\end{array}$ & $\begin{array}{l}\text { 5-7 times as long as calyx, } \\
\text { distinctly thickened above. }\end{array}$ \\
\hline Sepals & $\begin{array}{l}7 \times 3 \mathrm{~mm} \text { at anthesis, elliptic } \\
\text { to broadly lanceolate, acute } \\
\text { with broad scarious margins; } \\
\text { 1-nerved, midvein thickened } \\
\text { and indurate in fruit others } \\
\text { not elevated, especially } \\
\text { towards base. }\end{array}$ & $\begin{array}{l}6-9 \mathrm{~mm} \text { at anthesis, broad } \\
\text { and lanceolate, elevated 1- } \\
\text { veined, and c. } 10 \text { thin vein. }\end{array}$ & $\begin{array}{l}\text { 5-6 x 1.8-2 mm at anthesis, } \\
\text { narrowly lanceolate, with } \\
\text { narrow scarious margins, } \\
\text { caudate; 3-nerved, not } \\
\text { indurate }\end{array}$ \\
\hline Petals & $\begin{array}{l}2 / 3 \text { as long as sepals, elliptic- } \\
\text { oblong, obtuse }\end{array}$ & $\begin{array}{l}\text { slightly } 2 \times \text { longer than the } \\
\text { sepals, oblong to } \\
\text { subspathulate or broadly } \\
\text { spathulate to oblong or } \\
\text { cuneate at base }\end{array}$ & $\begin{array}{l}\text { as long as sepals or rarely } \\
\text { exceeding, spatulate }\end{array}$ \\
\hline Anthers & Yellow & Yellow & Mauve \\
\hline Capsule & $\begin{array}{l}2 / 3 \text { as long as sepals, } \\
\text { ellipsoid }\end{array}$ & Ovoid, at most equal to sepal & $\begin{array}{l}\text { as long as sepals (sub } \\
\text { mature) }\end{array}$ \\
\hline Seeds & $0.5-0.6 \mathrm{~mm}$ & $0.8-1.1 \mathrm{~mm}$ & c. $0.5 \mathrm{~mm}$ \\
\hline
\end{tabular}

3.3.4. Moenchia graeca Boiss. \& Heldr., Diagn. Ser. 2(1): 91 (1853). [Fig.7, 8]

Type: Many syntypes from Greece.

Small annual herbs. Stems 2-15 cm, usually unbranched. Basal leaves oblanceolate and more or less withered at anthesis; cauline leaves 2-4 pairs, 4-14 x 1.5-2.5 mm, linear-elliptic, subacute. Inflorescence 1-5 flowered. Bracts elliptic with broad scarious margins. Pedicels 2-8 times as long as calyx, distinctly thickened above. Sepals 7 x $3 \mathrm{~mm}$ at anthesis, elliptic to broadly lanceolate, acute with broad scarious margins; midvein thickened and indurate in fruit. Petals shorter than sepals, elliptic-oblong, obtuse. Capsule shorter than sepals. Seeds 0.5-0.6 mm.

\section{Examined specimens}

Denizli: Baymanlı mevkii, 700m, 18 v 1973, E.Tuzlacı (ISTE 24867!).

İzmir: Karaburun, Akdağ, 550m, 11 iv 1982, Sauer, E. et al. (IZEF 947!).

Manisa: Kula, lav akıntıları, 700m, 21 vi 1965, M.J.E.Coode ve B.MG.Jones (E !). 


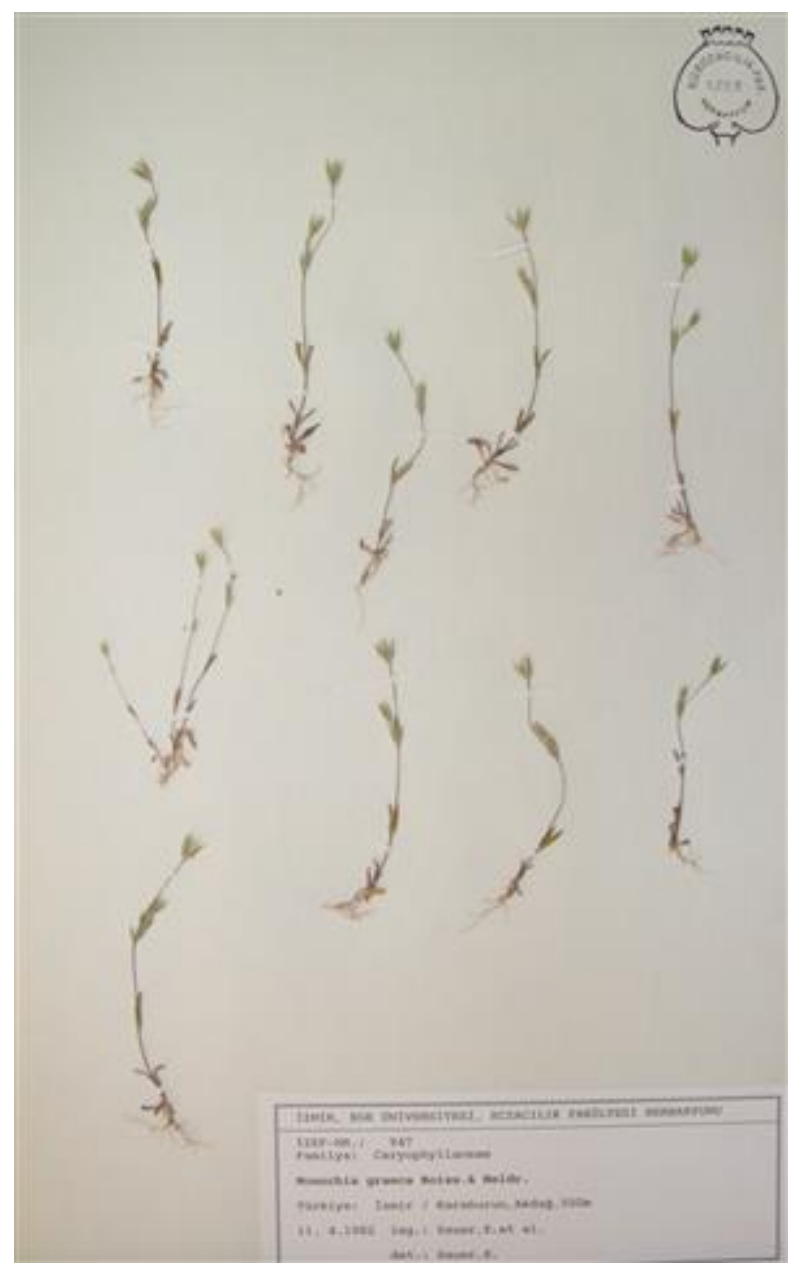

Figure 7. A Herbarium sheet

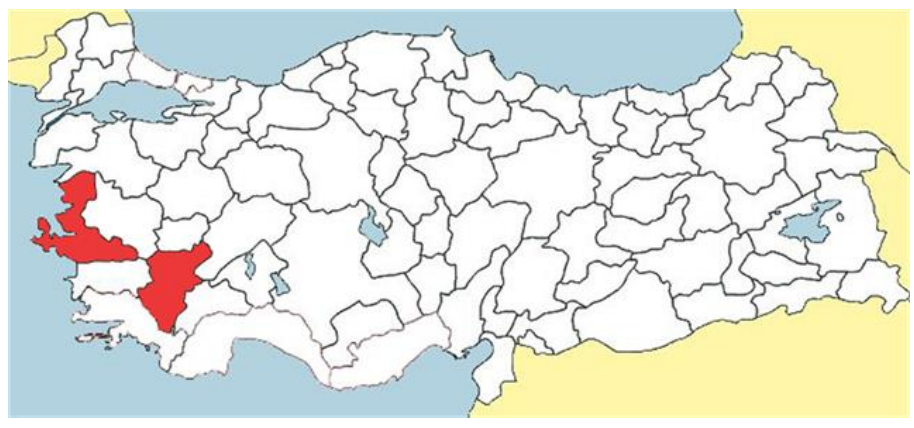

Figure 8. Distribution map

M. graeca is a little known species in Turkey. It is probably present in all Aegean and Mediterranean coasts. It is easily overlooked because of its small structure, short corolla, and early flowering phase.

\subsubsection{Moenchia erecta (L.) P.Gaertn., B.Meyer \& Scherb., Fl. Wett. 1: 219 (1799).}

Small annual herbs. Stems $2.5-15 \mathrm{~cm}$, glabrous, usually glaucous. Leaves somewhat petiolated at the base, 5$18 \mathrm{~mm}$, linear to oblanceolate; cauline leaves sessile, 3-12 mm, linear to lanceolate, ascending, subrigid, acute. Inflorescences lose, a few-flowered. Bracts ovate-lanceolate with broad scarious margin. Pedicel unequal, longest pedicel 25-65 mm. Sepal 4; 4.5-7 mm, 3-veined, lateral faint, linear-lanceolate with broad scarious margin. Petals 4; linear-lanceolate, white, shorter than sepals. Stamens 4 or 8 . Stillus 4 (-5). Capsule equalling or slightly longer than sepals, more or less cylindric. Seeds reddish-brown, finely tuberculate, $0.5-0.7 \mathrm{~mm}$ diam.

a. var. erecta $[$ Fig.9,10]

Type: Lectotype, Herb. Linn. No. 177.3 (LINN).

Syn.: Sagina erecta L., Sp. Pl.: 128 (1753). Cerastium tenue Viviani, Fl. Cors. 7 (1824). M. quaternella Ehrh., Beitr. Naturk., 2: 180 (1788) nom. illeg. M. glauca Pers., Syn. Pl., 1: 153 (1805) nom. illeg. Cerastium quaternellum Fenzl, Verbreit. Alsin.: 56 (1833) nom. illeg. Cerastium glaucum Gren., Mém. Soc. Émul. Doubs, 1: 47 (1841). Cerastium erectum (L.) Cosson \& Germain, Fl. Env. Paris, éd. 1: 30 (1845).

Longest pedicel 25-55 mm. Sepal 4; 6-7 mm, glabrous, veined. Petal 1/2 to 2/3 as long as sepals, usually subdentate. Stamens 4. Capsule equalling or slightly exceeding sepals.

\section{Examined specimens}

Edirne: Keşan-Enez yolu, Kılıçköy'den 1.5km önce, -merada otlu çukurlar ve tepecikler üzerinde- 18 iv 1992 , A.Byfield B 22, (ISTE 64848!). 
İstanbul: Kısırkaya köyü ile plaj arasında, yamaçlarda, iv 1992, A.Byfield B114 (ISTE 64884!). Pendik, Kurtköy, çataldağı, çayırlıklar ve kıraç kayalıklar, 30 iv 1995, A.J.Byfield B 2145 (ISTE 69498!). Yeşilköy havalimanı, 15 km W (E5), yol kenarı çayır, genç Pinus plantasyonunda, 9 iv 1972, I.Kukkonen (ISTF 25907!).

İzmir: Yamanlar dağı, Karagöl'den Maninsa geçidine doğru ormanlık dağ yolu, 9 vii 1966, R.Alava 5104 ve G.Bocquet (E!).

Muğla: Çine'den Yatağan'a giderken 15.km, nehir üstlerinden kayalık yamaçlarda, kış aylarında oluşan su sızıntıları arasında, otlakta, 24 iv 1992, A.Byfield B44 (ISTE 64870!). Marmaris körfezinde, Yıldız adası, 150m, iii 1993, A.Byfield B 134 (ISTE 65739!).

Tekirdağ: İnecik-Yenice, Yenice'den 7.2km önce, 18 iv 1992, A.Byfield B 10 (ISTE 64836!).

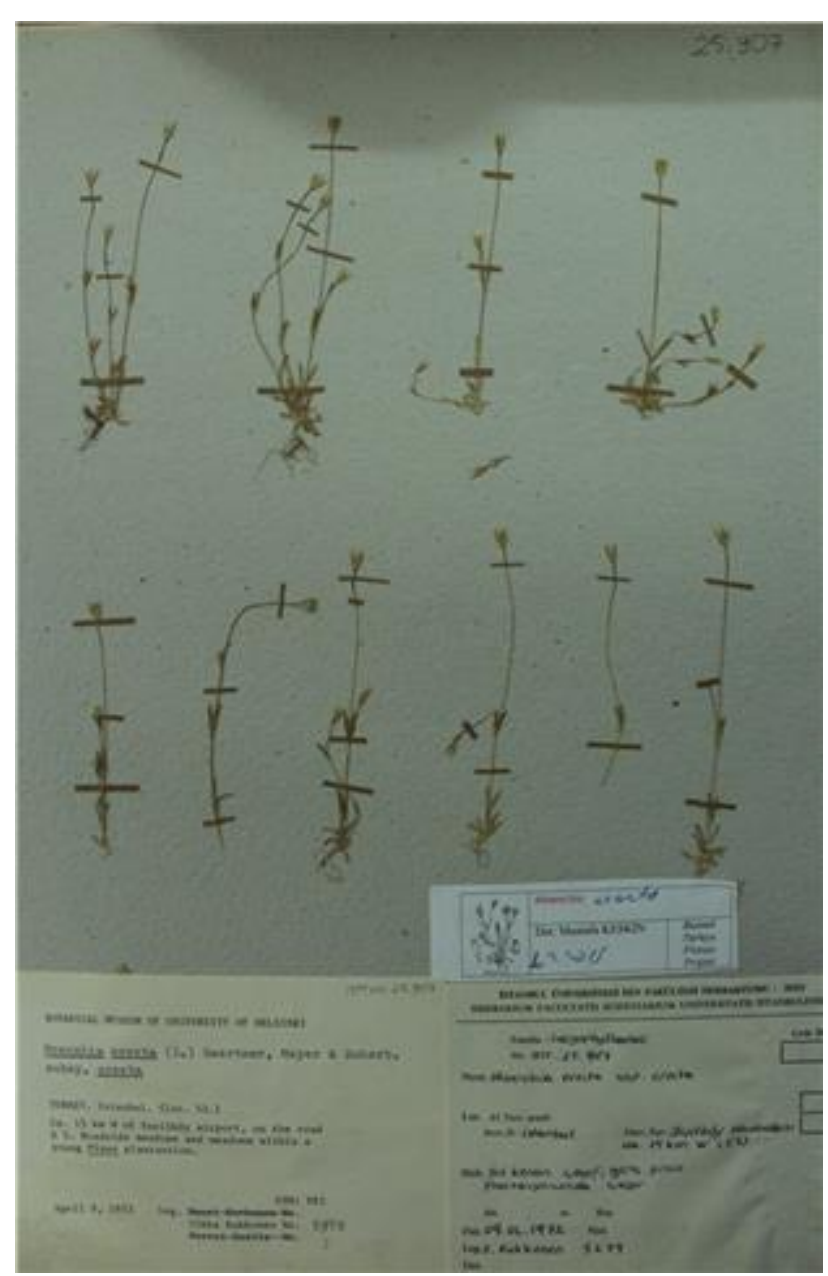

Figure 9. A Herbarium sheet

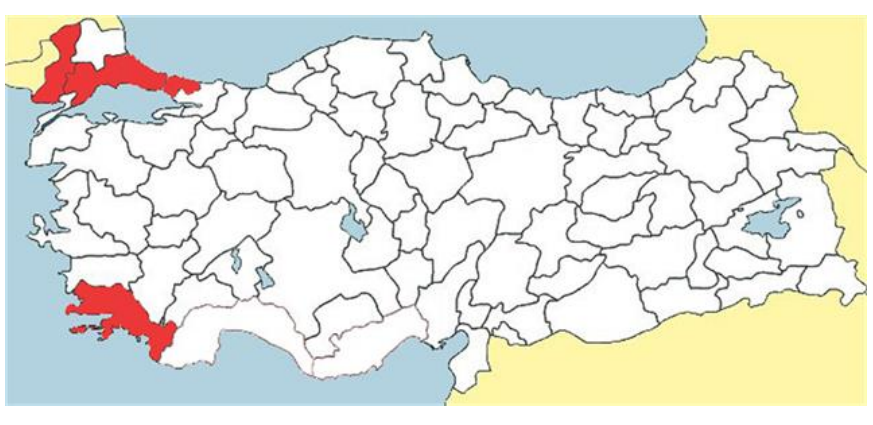

Figure 10. Distribution map

b. var. octandra (Ziz ex Mert. \& W.D.J.Koch) M.Keskin stat. nov. [Fig.11, 12]

Type: Described from Germany.

Syn.: M. octandra Rchb., Fl. Germ. Exc.: 703 (1832). M. octandra (Ziz ex Mert \& W.D.J.Koch)J.Gay, Perrym. Pl. Phan. Frejus 55 (1833). M. quaternella Ehrh. B. octandra Moris, Fl. Sard., 1: 269 (1837). M. quaternella Ehrh. subsp. octandra (Rchb.) Nyman, Consp. Fl. Eur. 111 (1878). Sagina octandra Ziz ex Mert. \& W.D.J.Koch, Deutschl. Fl., ed. 3, 1: 864 (1823). M. erecta subsp. octandra (Ziz.)Coutinho, Fl. Portugal: 211 (1913).

Longest pedicel 50-65 mm. Sepal 4; 4-5 mm, glabrous, veined, usually subdentate. Petal 4, about as long as sepals or a little shorter. Stamens 8 . Capsule scarcely equalling the sepals.

Examined specimens

İstanbul: Adalar: Büyükada, adanın arka tarafı, çayırlık, 17 iv 2017, M.Keskin 6914a!. Adalar: Burgazada, 18 v 1893 , Aznavour (E !). Sancaktepe, Turkuaz su fabrikası yakınları, Fabrikalar alanı, çayır, 15 iv 2017, M.Keskin 6814!. Zincirli to Şişli, 6 iv 1898, Aznavour (E !).

İzmir: Emiralem, Manisa ile Menemen aras1, 6 iv 1974, K.Lewejohann (Tu-74-203) ve F.Holtz (E !). 

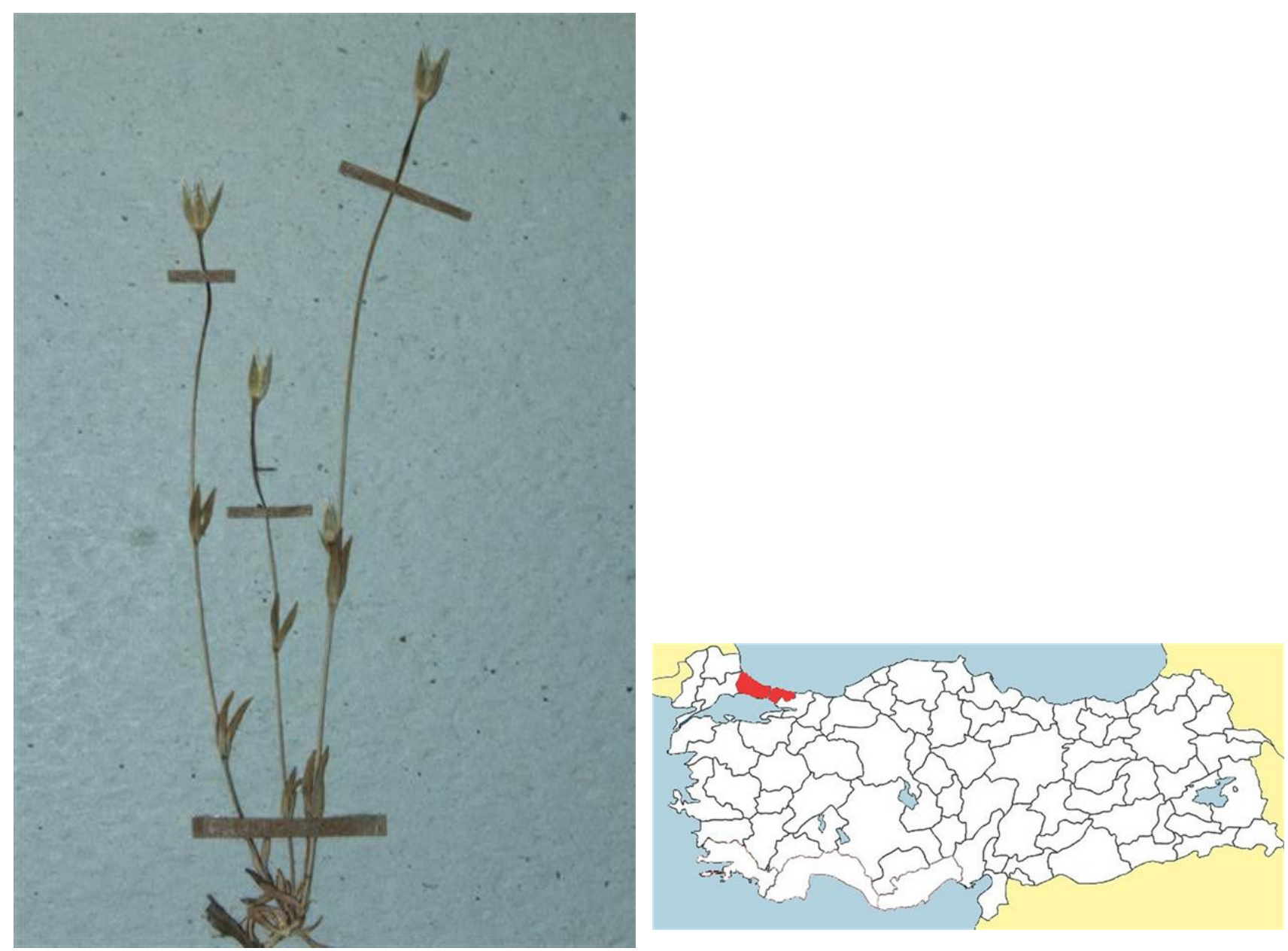

Figure 11. A Herbarium sheet

Figure 12. Distribution map

M. erecta is generally similar to the $M$. graeca. It is easily separated from the thickened pedicels, stamens with small numbers, and 4-part flowers. This species probably has a wider distribution in Turkey.

\section{Conclusions and discussion}

Although Moenchia is similar to Cerastium in general structure, it is separated from it by whole petal and calyx vein with prominent back.

In this study, it is reported to six Moenchia taxa in Turkey. Moenchia is not a widely known genus in Ankara province. Although there are many flora articles, this genus has never been reported [9, 10, 11, 12, 13, 14, 15]. One of them is the new species as M. akmanii M.Keskin. Although it looks like M. mantica in terms of appearance, its basic taxonomic characters are close to the M. graeca.

A new identification key to the species of Moenchia has been organized in Turkey. All herbarium records and distribution maps of the studied species have been prepared.

\section{Acknowledgments} foundation..

I would thank them for the fact that some herbarium visits for this study are supported by the ANG

\section{References}

[1] Strid, A. (1997). Moenchia L. In K. Tan \& A. Strid. (Eds.), Flora Hellenica (vol.1: 214-215). Koeltz Scientific Books, D-6240 Königstein/Federal Repubklic of Germany.

[2] Keskin, M. (2012). Moenchia L. In A. Güner \& al. (Eds.), Türkiye Bitkileri Listesi (Damarlı Bitkiler) [A Checklist of the Flora of Turkey (Vascular Plants)]. Nezahat Gökyiğgit Bahçesi ve Flora Araştırmaları Derneği Yayını. (pp. 348-349). İstanbul. (in Turkish). 
[3] Boissier, E. (1867). Moenchia L. Flora Orientalis: sive, Enumeratio plantarum in Oriente a Graecia et Aegypto ad Indiae fines hucusque observatarum (vol.1: 711-712). Genevae.

[4] Cafferty, S. \& Jarvis E. C. (Eds.). (2004). Typification of Linnaean plant names in Caryophyllaceae. Taxon 53 (4): 1049-1054. https://doi.org/10.2307/4135573.

[5] Clapham, A. R. (1964). Moechia L. In T. G. Tutin \& al. (Eds.), Flora Europaea (vol. 1: 145-146). Cambridge at the University Press.

[6] Cullen, J. (1968). Moenchia L. In P.H. Davis (Ed.), Flora of Turkey and The East Aegean Islands (vol. 2: 87-89). Edinburgh University Press.

[7] Ehrhart, F.J. (1778). Beiträge zur Naturkunde, und den damit verwandten wissenschaften, besonders der botanik, chemie, haus- und landwirthschaft, arzneigelahrtheit und apothekerkunst. (vol.2: 177). Hannover \& Osnabrück.

[8] Strid, A. (1991). Moenchia L. In K. Tan \& A. Strid. (Eds.), Mountain Flora of Greece (vol.2: 122). Edinburgh University Press.

[9] Yeşilyurt, E. B., Kurt, L. \& Akaydın, G. (2008). A Study on Flora of Hacıkadın Valley (Ankara/Turkey). Biological Diversity and Conservation 1/2: 25-52.

[10] Aslan, S. \& Vural, M. (2009). Flora of Kıbrıs Köyü Valley (Mamak-Ankara, Turkey). Biological Diversity and Conservation 2/3: 34-64.

[11] Karaman-Erkul, S. \& Aytaç, Z. (2011). Flora of Duatepe and its environment (Polatl1- Ankara/Turkey). Biological Diversity and Conservation 4/1: 17-29.

[12] Çakır, E. G., Akdoğan, G. E. \& Meral, G. (2015). The flora of Sarıçalı Mountain and arounds (Nallıhan, Ankara/Turkey). Biological Diversity and Conservation 8/3: 267-289.

[13] Çakmak, M. H. \& Aytaç, Z. (2018). Urban vascular flora and ecological characteristics of Mamak District (Ankara/Turkey). Biological Diversity and Conservation 11/2: 123-131. 784491.

[14] Başköse, İ., Körüklü, S. T. \& Yapyak, A. E. (2020). The Plant biodiversity of Ankara University 10. yıl (Beşevler) campus. Biological Diversity and Conservation 13 / 1: 217-231. DOI: 10.46309/biodicon.2020.

[15] Çelik-Gündoğmuş, İ. \& Ekici, M. (2021). Flora of Yassıhöyük (Gordion) (Polatl1/Ankara/Turkey). Biological Diversity and Conservation 14/1: 53-68. DOI: 10.46309/biodicon.2021.830260. 